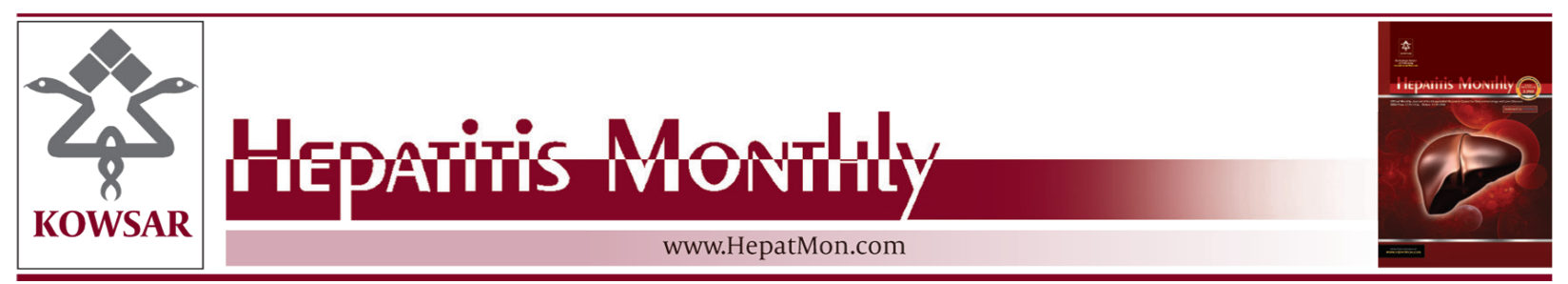

\title{
Psychometric Properties of a Standardized Questionnaire of Knowledge, At- titude, and Practice of Iranian Medical Specialists about Viral Hepatitis
}

\author{
Shahin Ghasemi ${ }^{1}$, Ali Kabir ${ }^{2,3,}$, , Mojtaba Ansari Jafari ${ }^{4}$, Mohammad Jalali ${ }^{5}$, Afshin Amini ${ }^{6}$, \\ Amir Hossein Faghihi-Kashani ${ }^{7}$, Seyed Moayed Alavian ${ }^{8}$ \\ ${ }^{1}$ Rasoul Akram Hospital, Tehran University of Medical Sciences, Tehran, IR Iran \\ ${ }^{2}$ Department of Epidemiology, Faculty of Public Health, Shahid Beheshti University of Medical Sciences, Tehran, IR Iran \\ ${ }^{3}$ Center for Educational Research in Medical Sciences, Tehran University of Medical Sciences, Tehran, IR Iran \\ ${ }^{4}$ Department of Nuclear Medicine, Shahid Beheshti University of Medical Sciences, Tehran, IR Iran \\ ${ }^{5}$ Department of Cardiology, Alborz University of Medical Sciences, Karaj, IR Iran \\ ${ }^{6}$ Imam Hossein Hospital, Faculty of Medicine, Shahid Beheshti University of Medical Sciences, Tehran, IR Iran \\ ${ }^{7}$ Department of Gastroenterology and Hepatology, Tehran University of Medical Sciences, Tehran, IR Iran \\ ${ }^{8}$ Gastroenterology and Hepatology Department, Baqyiatallah Research Center for Gastroenterology and Liver Diseases, Tehran Hepatitis Center, Tehran, \\ IR Iran \\ * Corresponding author: Ali Kabir, Department of Epidemiology, Faculty of Public Health, Shahid Beheshti University of Medical Sciences, Tehran, IR Iran. \\ Tel.: +98-2144468645, Fax:+98-2144476796, E-mail:aikabir@yahoo.com
}

\section{A B S T R A C T}

Background: Good knowledge, attitude and practice (KAP) of the physicians allow them to handle their patients in such a way that they prevent themselves from contracting, and their patients from spreading, the infection. However, the Iranian standardized KAP questionnaire of physicians about viral hepatitis is not available. So, we developed a standard questionnaire.

Objectives: The purpose of this study was to provide a standard questionnaire as a basic tool for assessment of the present situation of the KAP of clinicians. It can also be used for evaluating educational programs and interventions on physicians in addition to any trends in their KAP about viral hepatitis.

Patients and Methods: In order to design and standardize a 29-item self-administered questionnaire, we developed a cross sectional pilot study on 60 Iranian physicians. Ten experts in the field of liver diseases and/or designing the questionnaire answered questions about its validity. Cronbach's Alpha (on 60 physicians that participated in a congress) and factor analysis (on 370 persons; participants of two viral hepatitis congresses in Tehran and Zanjan and physicians of two university hospitals in Ahvaz) were used in the analysis.

Results: Reliability was 0.7 according to Cronbach's Alpha score. Face validity was higher than $80 \%$. Content validity of the whole parts of the questionnaire was $96.25 \%$ for clarity, $91.56 \%$ for relevancy, $96.25 \%$ for simplicity and $98.44 \%$ for consistency of each question with the questions' set. Factor analysis showed that 13 components account for $67.4 \%$ of the total variance.

Conclusions: This study provided evidence that our questionnaire is a feasible, valid and reliable measure of physicians' KAP status in Iran. The factor analysis did not reveal a strong cluster structure. This questionnaire should be interpreted as a one-dimensional element by the sum of all items, rather than a multi-dimensional instrument.

Keywords: Hepatitis; Questionnaires; Validation Studies; Reliability; Physicians

Copyright (ㅇ 2012, Kowsar Corp.; Published by Kowsar Corp.

Article type: Research Article; Received: 08 Aug 2012, Revised: 07 Sep 2012, Accepted: 27 Sep 2012; DOI: 10.5812/hepatmon.7650

Implication for health policy/practice/research/medical education:

This study is of importance for determining the situation of knowledge, attitude and practices (KAP) of Iranian physicians about viral hepatitis B and C, is useful in determining the trend of changes in their KAP by repeating the study and comparing the results, in designing further related studies in this field, and in designing interventions and assessment of implementation of new strategies for improvement the KAP of clinicians about viral hepatitis by either policy makers in Ministry or clinical researchers in the field. Moreover, revising more qualified questionnaire in this field can be done using this basic questionnaire. 
Please cite this paper as:

Ghasemi Sh, Kabir A, Ansari Jafari M, Jalali M, Amini A, Faghihi-KashaniAH, et al. Psychometric Properties of A Standardized Questionnaire of Knowledge, Attitude, and Practice of Iranian Medical Specialists about Viral Hepatitis. Hepat Mon. 2012:12(12):e7650. DOI: 10.5812/hepatmon.7650

Copyright (C) 2012, Kowsar Corp.; Published by Kowsar Corp

This is an Open Access article distributed under the terms of the Creative Commons Attribution License (http://creativecommons.org/licenses/by/3.0), which permits unrestricted use, distribution, and reproduction in any medium, provided the original work is properly cited.

\section{Background}

Hepatitis B and C virus (HBV and HCV) are among bloodborne diseases that still pose significant health problems $(1,2)$ which are easily preventable. Prevalence of HBV and HCV in the Iranian general population is $2.14 \%$ (3) and $0.16 \%$ (4), respectively. Physicians will visit some cases with HBV and HCV during their careers. Therefore, they should be aware of the principal questions patients are likely to ask them about their diseases: epidemiology of the disease, important risk factors and transmission routes, specifically the ones they encountered the most when in practice. It is important the physicians know a great deal about these diseases and use this knowledge when treating patients infected with HBV and HCV. It is the physician's attitude and commitment to strong awareness of these diseases that will assist them in teaching their patients and themselves how to prevent the spread of the infection. On the other hand, physicians need to provide their expertise in treating the infected cases. Such a questionnaire could assist health care providers in order to evaluate the adequacy of teaching programs for physicians and evaluate the necessity of retraining. Previous studies revealed that there is an urgent requirement for greater focus on viral hepatitis on the part of Iranian surgeons (5) and dentists (6-8). These previous studies have shown that the knowledge, attitude and practice of Iranian surgeons and dentists are not sufficient and we would require to focus on areas of their weakness and provide education and specific programs to remedy the situation. To evaluate both the areas of weaknesses and assess the need for remedial education, KAP surveys should be performed. Currently, an Iranian standardized questionnaire of knowledge, attitude and practice (KAP) of physicians about viral hepatitis is not presented. Similar questionnaires are also infrequently seen and used in the Middle East region in the field of liver diseases (9-14). Therefore, it is essential that the KAP status of physicians is assessed (and screened) by a standardized questionnaire regarding their KAP with respect to viral hepatitis. Such a questionnaire could be used as the best way to check the awareness, attitude and good practice toward HBV and HCV in physicians. In addition, it can be used by community programmers and policy makers of health-related issues in order to evaluate the requirements for educational programs and potentially necessary interventions.

\section{Objectives}

This article reports the development of an Iranian KAP questionnaire about $\mathrm{HBV}$ and HCV and the results of its psychometric testing among physicians with different specialties from different cities in Iran. The marked increase in the survival of patients with viral hepatitis due to medication (15) and liver transplantation (16), together with the effect of the disease on social and family life (17), have led researchers to develop a questionnaire that can be used as a standard tool for further research.

\section{Patients and Methods}

\subsection{Study Population}

We circulated questionnaires among participants in two viral hepatitis congresses: in Tehran (capital city of Iran, a national congress) and in Zanjan (in western Iran, a regional congress) and also among physicians of two university hospitals in Ahvaz (located in southern part of Iran). Participants returned 370 (82\%) of the 450 distributed questionnaires. We excluded the results of one questionnaire due to the absence of more than $50 \%$ of the data. In this cross sectional study, we considered dentists, general practitioners (GPs), paraclinicians (laboratory specialists, pathologists, anesthesiologists, radiologists, and parasitologists), surgeons (gynecologists, general surgeons, and orthopedic surgeons) and internists (cardiologists, pediatricians, dermatologists, specialists in internal medicine, infectious disease, and emergency medicine) as different groups. This project consisted of different steps for producing and standardizing a questionnaire about KAP of physicians as it relates to hepatitis $B$ and $C$. Further to designing the questionnaire as a first step, we conducted a cross sectional study of 60 doctors with different specialties, and had them answer the questions in a self-administered manner. Second step was followed by a reliability analysis and factor analysis for reducing the items as much as possible. As a third step, we had 10 experts ensure the validity of the questionnaire.

\subsection{Designing and Standardizing the Question- naire}

A 29-item self-administered questionnaire assessing risk of transmission; seroconversion rates; the actual prevalence of HBV and HCV in Iran; vaccination against 
$\mathrm{HBV}$; use of double gloves and protective eyewear; rate of needle stick injury and its reporting; checking the status of viral hepatitis; the use of disposable syringes and how they are discarded; and post-exposure prophylaxis had been designed. The questionnaire used the Likert scale; yes/no and a few open-ended questions, in addition to some demographics (age, gender, specialty, number of hours spent at work per week).

\subsubsection{Item Generation}

After a thorough search through the available litera- ture, we planned two focus group discussions and one expert panel in order to design flowchart (Appendix 1) and for characterizing the main domains of our KAP survey. Then, we detailed our main domains to some questions. Experts in the field of methodology (epidemiologists; specifically one who was the specialist in designing the questionnaire), a psychiatrist, a specialist in Community Medicine, an infectious diseases expert and gastroenterologists were most important part of our expert panel and focus group discussions.

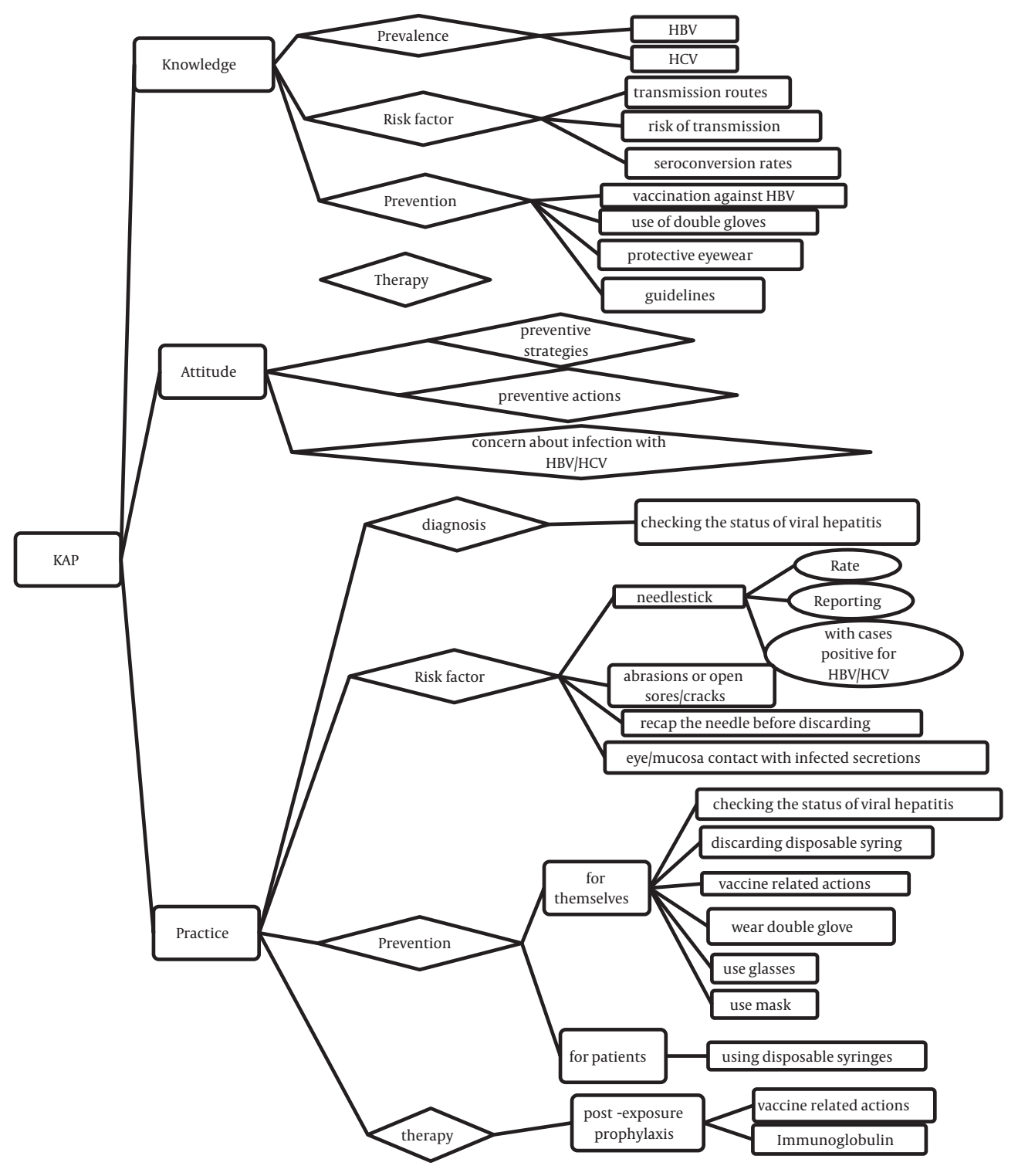

Appendix 1. Flowchart of the Questionnaire 


\subsubsection{Item Modification}

According to the expert methodologists' opinions, the structure and the content of some questions had been altered. A pilot study on 60 Iranian physicians with various specialties (participating in the scientific national congresses) was performed to assist in order to modify the structure and content of the primary questionnaire. Answers to each question were also revised according to the different answers to questions in the pilot study.

\subsubsection{Item Reduction (Factor Analysis)}

Factor analysis provides an enhanced understanding of which variables forms a "relatively coherent subset, independent of others" (18). We performed this analysis on 370 subjects to realize if our most important domains (knowledge, attitude and practice) were categorized by this analysis in the same pattern we first categorized them. We planned to confirm the primary flowchart ( Appendix 1 ) of the questionnaire in this approach.

\subsubsection{Item Standardization}

\subsubsection{Reliability}

Internal consistency reliability (Cronbach's Alpha), measuring the extent that the questions in each domain and all three main parts (knowledge, attitude and practice) tap a particular concept (19) was determined according to the pilot study on 60 physicians with various specialties.

\subsubsection{Face Validity}

A separate sample of ten experts in the field of liver diseases and/or designing the questionnaire reviewed the questionnaire and answered the question: "How well do you think the questionnaire measures knowledge, attitude and practice of a physician about hepatitis B and C"? They responded using a 5-point Likert scale from 1 (not at all) to 5 (very well).

\subsubsection{Content Validity}

The content validity of the final questionnaire was determined according to the clarity, relevancy, simplicity, and consistency of each question with the questions set from 10 experts in the field of liver diseases ( 5 persons) and methodologists (5 persons). They examined the questionnaire for important omissions or inappropriate choice of items. To decrease the prestige bias, demographic variables were inserted at the end of questionnaire. For quality assurance, there was also guidance provided for some questions so the recipient would know how some specific questions should be answered.

\subsection{Demographic Variables}

Age, gender, specialty, place of work, and the quantity of activity in medical practice were among our demographic variables.

\subsection{Data Analysis}

We calculated the internal consistency of the questionnaire using Cronbach'sAlpha coefficient. Factor analysis was done for data reduction and grouping the related variables in conceptually similar and statistically related groups. The extraction method was aprincipal components analysis, the varimax rotation method, and we extracted factors based on an Eigenvalue larger than 1. Kaiser-Meyer-Olkin measure of sampling adequacy and Bartlett's test of sphericity were used and the cut off point for loading on each factor was 0.3. We used mean \pm SD for expressing quantitative variables and correlation test with Pearson coefficient for assessing the relationship between these variables. The analysis was done by SPSS 13 (SPSS Inc. Chicago, Illinois, USA) and Excel software. The authors considered differences and correlations, with $P$ $<0.05$ being statistically significant. For calculating the Item Content Validity Index (I-CVI), the average of our experts which believed that item is desirable/completely desirable was calculated and expressed as percent. The Scale Content Validity Index (S-CVI) for clarity, relevancy, simplicity, and consistency was calculated as the average of items which our experts believed that were desirable/ completely desirable.

\subsection{Ethics}

All subjects signed an informed written consent before participating in the study. The ethics committee of the Baqyiatallah Research Center for Gastroenterology and Liver Disease from Baqyiatallah University of Medical Sciences, Tehran, Iran approved the study proposal.

\section{Results}

The final questionnaire consisted of four parts (Appendix ${ }^{2}$ ): 1 . Seven questions about knowledge, 2. Eighteen questions about practice, 3. Four questions about attitudes, and 4. Seven questions about demographic variables.

\subsection{Demographic Characteristics of Study Partici- pants}

Generally, most of the participants were young females. The number of males was 161 (43.6). They had an age average (SD) equal to 40.8 (8.9) and age range between 25 and 79 years, a median duration in medical practice of 12 (range: 0.3 to 50 ) years, and a median weekly medical practice of 35 (range: 2 to 120 ) hours. Of the participants, $36.3 \%$ were employees of university hospitals and clinics of the Ministry of Health, $39.8 \%$ worked in private practice, and $23.9 \%$ worked in both private practice and as employees of clinics and university hospitals. 


\subsection{Psychometric Properties}

\subsubsection{Reliability}

Cronbach's Alpha score, measuring the internal consistency of questions was 0.7. Its value in each domain has been shown in ( Table 1 ).

Table 1. Internal Consistency of Different Domains

\begin{tabular}{ll}
\hline Domain & Cronbach's Alpha \\
\hline Knowledge & 0.65 \\
Practice & 0.75 \\
Attitude & 0.67 \\
\hline
\end{tabular}

\subsubsection{Face Validity}

All experts rated the question 4 or higher except two persons, producing an overall mean of 4 .

\subsubsection{Content Validity}

The characteristics of the content validity of the whole parts of the questionnaire were clarity: $96.25 \%$, relevancy: 91.56\%, simplicity: $96.25 \%$ and consistency of each question with the questions' set: $98.44 \%$. Percent of content validity according to different domains has been shown in ( Table 2 ).

Table 2. Percentage of Content Validity According to Different Domains

\begin{tabular}{lllll}
\hline Domain & Clarity & Relevancy & Simplicity & Consistency \\
\hline $\begin{array}{l}\text { Knowl- } \\
\text { edge }\end{array}$ & 98.57 & 88.57 & 98.57 & 95.71 \\
Practice & 95.24 & 92.38 & 96.19 & 99.05 \\
\hline Attitude & 97.5 & 92.5 & 92.5 & 100 \\
\hline
\end{tabular}

\subsection{Factor Analysis}

The sample size for the factor analysis was 29 items with 370 subjects. KMO measure of sampling adequacy was 0.557 was considered adequate for the factor analysis, and Bartlett's test of sphericity also demonstrated a satisfactory suitability of the data to factor analysis $(P<0.001)$ which shows that our variables are related and therefore suitable for structure detection. Extraction communalities are estimates of the variance in each variable accounted for by the components. Our communalities were all above 0.58 and most of them above 0.64. So, all are high which indicates that the extracted components represent the variables well and we do not need to extract another component. A loading cutoff of $>0.30$ was adopted, and 13 factors were extracted. Each factor explained 2.98 to $10.39 \%$ of the total variance, and $67.4 \%$ of the variance was explained by these 13 factors, revealing a weak factor structure. We repeated factor analysis by recoding all variables to three options (even for dichotomized variables). The results did not change significantly.

\subsection{Findings of the Pilot Study About Main Out- comes}

Results are completely explained in a separate paper (20).

\section{Discussion}

Although validation studies could be considered as challenging cases to conduct, their results might be considered worthwhile in various cultures. The first benefit is that they provide standard health measures which allow for health status comparisons between countries. Secondly, they provide validated instruments to monitor population health, to estimate the burden of disease, and to investigate outcomes in clinical practice and to evaluate treatment effects (21). This study provided evidence that our questionnaire is a valid measure of physicians' KAP status in Iran. Questionnaire was designed to be a self-administered questionnaire, but it could be completed through an in-person interview, computerized administration, or by telephone after small validation studies. Furthermore, the study used a relatively large sample of diverse physicians as defined by different age groups, gender, specialties, expertise, cities of practice, duration of practice and location of practice (clinic and/or hospital, and private and/or governmental). Therefore, the standardized questionnaire acquired from this study could reliably be used for all Iranian physicians. In general, all psychometric tests of the questionnaire showed satisfactory results. Reliability of the questionnaire as measured by the Cronbach's Alpha coefficient for all three scales and all parts at once exceeded the recommended level. With I-CVI and S-CVI, all percentages were higher than $80 \%$ which are considered as the minimum acceptable for a new tool (22-25). Therefore nearly all of them were acceptable and such a questionnaire can be used in similar situations (Iranian physicians about viral hepatitis). It may also be used in other Iranian cases which do not have significant differences affectingthe validity of this questionnaire. The factor analysis did not reveal a strong cluster structure, suggesting that the questionnaire should be interpreted as a one-dimensional element by the sum of all items, rather than as a multi-dimensional instrument. In general, the questionnaire should be interpreted with the sum of all items rather than as isolated items or clusters of questions. Factor analysis confirmed that there are not limited numbers of questions that can explain most of variance of the questionnaire. Thirteen factors explained $67 \%$ of the variance and shows that we have truly split the different parts of KAP into subtitles with the least overlap. Factors were related to the same parts of KAP. The significance level of Bartlett's test was less than 0.05 in 
our study which indicates that a factor analysis may be useful with our data. Few questionnaires of this type have published data on their validity and reliability and we did not find a similar one to which we could compare our results. So, we compared our findings with standard indices like 0.7 for Cronbach's Alpha and $80 \%$ for CVI. Obviously, we could have a translation of an existing questionnaire. However, since translating a questionnaire is strenuous and a translation might not be suitable for all subgroups in view of medical terminology and practices as well as general knowledge about hepatitis differ among populations. One limitation of this study is that although our research began with careful operationalization of the domain of viral hepatitis-related knowledge, attitude and practice of physicians with a rational item selection phase, the resulting measure cannot be considered a comprehensive measure of viral hepatitis-related KAP. Although the questionnaire emphasizes some more important parts, it does not comprehensively assess all risk factors in details. Moreover, the measure does not attempt to assess knowledge regarding the natural history, clinical course, or treatment of viral hepatitis. Thus, in applications or settings where a more comprehensive assessment is needed, we recommend expenditure of a longer and in more detailed questionnaire. However, it could be mentioned that longer questionnaires have a lower validity and reliability because of a diminution in the attention of the responders. Although our study did not provide evidence for test-retest reliability, responsiveness to change or other psychometric tests, the findings showed that this questionnaire is a reliable appraise for measuring physicians' KAP about viral hepatitis. Future studies could focus on other psychometric properties of the questionnaire and also on different applications of the questionnaire. In conclusion, although the present study does not provide evidence on test-retest reliability or on responsiveness, the findings however provide further evidence that this questionnaire about viral diseases is feasible in Iran and could be used as a reliable and valid instrument for measuring KAP of physicians. It could be used in several academic research projects and further results from its validity are anticipated.

\section{Acknowledgements}

The authors would like to thank Dr Bita Behnava, Dr Hamid Reza Gilasi, Dr Seyed Mohammad Miri, Dr Hossein Alaie, and Dr Mostafa Alavi Moghadam for their collaborations and contributions to the study and also commenting and scoring questions in order to validating the questionnaire.

\section{Financial Disclosure}

None declared.

\section{Funding/Support}

None declared.

\section{Authors' Contribution}

None declared.

\section{References}

1. Nebbia G, Peppa D, Maini MK. Hepatitis B infection: current concepts and future challenges. QJM. 2012;105(2):109-13.

2. Sievert W, Altraif I, Razavi HA, Abdo A, Ahmed EA, Alomair A, et al. A systematic review of hepatitis $C$ virus epidemiology in Asia, Australia and Egypt. Liver Int. 2011;31(Suppl 2):61-80.

3. Alavian S, Hajarizadeh B, Ahmadzad-Asl M, Kabir A, Bagheri-Lankarani K. Hepatitis B virus infection in Iran: A systematic review. Hepat Mon. 2008;8(4):281-94.

4. Alavian S, Ahmadzad-Asl M, Lankarani K, Shahbabaie M, Ahmad A, Kabir A. Hepatitis C infection in the general population of Iran: A systematic review. Hepat Mon. 2009;9(3):211-23.

5. Moghimi M, Marashi SA, Kabir A, Taghipour HR, FaghihiKashani AH, Ghoddoosi I, et al. Knowledge, attitude, and practice of Iranian surgeons about blood-borne diseases. J Surg Res. 2009;151(1):80-4.

6. Mahboobi N, Agha-Hosseini F, Safari S, Lavanchy D, Alavian SM Hepatitis B virus infection in dentistry: a forgotten topic. J Viral Hepat. 2010;17(5):307-16.

7. Alavian SM, Mahboobi N, Savadrudbari MM, Azar PS, Daneshvar S Iranian dental students' knowledge of hepatitis B virus infection and its control practices. J Dent Educ. 2011;75(12):1627-34.

8. Alavian SM, Mahboobi N. Hepatitis B infection in dentistry setting needs more attention. Med Princ Pract. 2011;20(5):491-2.

9. Kamolratanakul P, Ungtavorn P, Israsena S, Sakulrarnrung R. The Influence of dissemination of information on the changes of knowledge, attitude and acceptance of hepatitis B vaccination among hospital personnel in Chulalongkorn Hospital. Public Health. 1994;108(1):49-53.

10. Sood A, Midha V, Awasthi G. Hepatitis C--knowledge \& practices among the family physicians. Trop Gastroenterol. 2002;23(4):198201.

11. Askarian M, Khalooee A, Emroodi NN. Personal hygiene and safety of governmental hospital staff in Shiraz, Islamic Republic of Iran. East Mediterr Health J. 2006;12(6):768-74.

12. Kagan I, Ovadia KL, Kaneti T. Physicians' and nurses' views on infected health care workers. Nurs Ethics. 2008;15(5):573-85.

13. Shiva F, Sanaei A, Shamshiri AR, Ghotbi F. Survey of needle-stick injuries in paediatric health personnel of 5 university hospitals in Tehran.J Pak Med Assoc. 2011;61(2):127-31.

14. Lahariya C, Khandekar J, Vachher AS, Pradhan SK. Physicians and communities knowledge and awareness about new vaccines in immunization program: a study to derive lesson for increasing uptake. Kathmandu Univ Med J (KUMJ). 2010;8(29):51-6.

15. Roche B, Samuel D. Hepatitis C virus treatment pre- and postliver transplantation. Liver Int. 2012;32(Suppl 1):120-8.

16. Buchanan C, Tran TT. Current status of liver transplantation for hepatitis B virus. Clin Liver Dis. 2011;15(4):753-64.

17. Alavian S, Kachuee H, Moghani Lankarani M, Assari S, Farmanara H. Marital Adjustment in Patients with Chronic Viral Hepatitis versus Healthy Controls. Iran J Psychiatry. 2006;1(4):153-7.

18. Kahn J. Factor Analysis in Counseling Psychology Research, Training, and Practice: Principles, Advances, and Applications. Couns Psychol. 2006;34(5):684-718.

19. Aday L, Cornelius L. Designing and conducting health surveys : a comprehensive guide. 2006; $\mathrm{p}$.

20. Kabir A, Tabatabaei SV, Khaleghi S, Agah S, Faghihi Kashani AH Moghimi M, et al. Knowledge, attitudes and practice of Iranian medical specialists regarding hepatitis B and C. Hepat Mon. 2010;10(3):176-82.

21. Montazeri A, Goshtasebi A, Vahdaninia M, Gandek B. The Short 
Form Health Survey (SF-36): translation and validation study of the Iranian version. Qual Life Res. 2005;14(3):875-82.

22. Boggess KA, Urlaub DM, Moos MK, Polinkovsky M, El-Khorazaty J, Lorenz C. Knowledge and beliefs regarding oral health among pregnant women. J Am Dent Assoc. 2011;142(11):1275-82.

23. Bowyer V, Sutcliffe P, Ireland R, Lindenmeyer A, Gadsby R, Graveney M, et al. Oral health awareness in adult patients with diabetes: a questionnaire study. Br Dent J. 2011;211(6):E12.

24. Salcedo-Rocha A, García-de-Alba-Garcia J, Velásquez-Herrera J,
Barba-González E. Oral Health: Validation of a questionnaire of self-perception and self-care habits in Diabetes Mellitus 2, hypertensive and obese patients. Med Oral Patol Oral Cir Bucal. 2011;16(6):834-9.

25. Defranc A, Van den Broucke S, Leroy R, Hoppenbrouwers K, Lesaffre E, Martens L, et al. Measuring oral health behaviour in Flemish health care workers: an application of the theory of planned behaviour. Community Dent Health. 2008;25(2):107-14. 